\title{
Artificial Neural Networks in Prediction of Patient Survival after Liver Transplantation
}

\section{Raji CG ${ }^{1^{*}}$ and Vinod Chandra SS $^{2}$}

${ }^{1}$ Department of Computer Science and Engineering, M.S University, Tirunelveli, Tamil Nadu, India

${ }^{2}$ Computer Centre, University of Kerala, Thiruvananthapuram, Kerala, India

*Corresponding author: Raji CG, Department of Computer Science and Engineering, M.S University, Tirunelveli, Tamil Nadu, India, Tel: 0462-2338632; E-mail: rajicg80@gmail.com

Received date: November 14, 2015; Accepted date: January 28, 2016; Published date: February 05, 2016

Copyright: (c) 2016 Raji CG, et al. This is an open-access article distributed under the terms of the Creative Commons Attribution License, which permits unrestricted use, distribution, and reproduction in any medium, provided the original author and source are credited.

\begin{abstract}
Predicting the survival of patients after liver transplantation is one of the challenging areas in the field of medicine. The ultimate curative treatment for the last stage liver disease is the liver transplantation. While going for any transplantation, everybody will think about the survival. This paper summarizes the prediction of survival of patients undergoing liver transplantation in both computing and clinical manner. We proposed an Artificial Neural Network model to define three month mortality of patients after liver transplantation using United Network for Organ Sharing dataset. We trained the data using Multilayer Perceptron Artificial Neural Network model using 10 fold cross validation and achieved an accuracy of $99.74 \%$. The comparison of our model was done with other Artificial Neural Network models with the help of various performance error measures. In order to ensure accuracy produced by the model, we also made comparison with existing models in the prediction of survival of patients after liver transplantation.
\end{abstract}

Keywords: Liver transplantation; Model for end stage liver disease (MELD); Survival prediction; Artificial neural network

\section{Introduction}

Transplantation of human liver is a curative treatment insisted for end stage liver disease [1]. The tremendous developments in the field of liver transplantation throw light to the humans which are in need of survival. The prediction of survival is an important factor used to determine the success of liver transplantation surgery. The surgical consequences in liver transplantation depend upon various factors like disease severity, availability of donor organs, immunosuppression and forecasting the survival. In medicine, the medical experts get the judgment of outcome of liver transplantation based on Model for End Stage Liver Disease (MELD) score [2].

As it follows the sickest first policy, the patients in the waiting list first will get more priority for liver transplantation than others [2]. MELD consists of three parameters namely Creatinine, Bilirubin and International Normalized Ratio (INR), out of which Creatinine is lower in females than males [2]. Another difficulty with MELD score is that Creatinine varies with body weight of the patient [2]. The liver patients are further ranked with donor's and recipient's age, medical urgency of recipient and the geographical proximity among donor and recipient. Because of the absence of more precise models, the medical experts still depends upon the traditional scoring system. The continuous exploration of more accurate survival models results in the introduction of Artificial Neural Networks (ANN). A large number of logistic regression models and conventional statistical techniques are also used for the prediction of survival of patients after LT. In order to overcome the problem with local minima and nonlinear problem solving, ANN features over rule the conventional statistical techniques and logistic regression models [3]. ANN models are nonlinear models which can solve the problems with conventional models and produce the results in high accurate manner [3].

\section{Background of existing solutions}

Researchers conducted studies with logistic regression models and ANN for the prediction of survival of patients after liver transplantation. In 1992, Doyle et al. [4] derived an expression using stepwise logistic regression analysis to predict the graft failure in liver recipients. They collected the data from Presbyterian University Hospital, Pittsburg, PA from January to August 1992 [4]. Labroc 1 software was used to evaluate their results. But that time they have only small amount of data to perform the study. So they failed to produce the accuracy in the prediction of survival of patients after liver transplantation [4]. In order to overcome the drawbacks in that study, the same team of researchers introduced a 10 feed forward back propagation neural network model to predict liver transplantation survival [5]. In that study, they used training and test sets with random sampling and explained the nonlinearity among variables [5]. Parmanto et al. [6] conducted a study with time series sequence of medical data with the help of recurrent neural networks using Back Propagation through Time (BPTT) algorithm.

They evaluated their results with 6-fold cross validation [6]. Cucchetti et al. [7] proposed an ANN model and proved that it is better than MELD. They conducted the experiment with 251 consecutive liver people with cirrhosis at liver transplantation unit, Bologna, Italy in 2006 [7]. Marsh et al. [8] presented a three layer feed forward fully connected ANN model to predict the survival analysis and time to recurrence of Hepato Cellular Carcinoma (HCC) after Orthotropic liver transplantation (OLT). They worked with data of 214 patients at the University of Pittsburgh Medical Center [8]. The researchers proved that female patients were having less risk of recurrence of HCC than males through this study [8]. Zhang et al. [9] 
Page 2 of 7

proposed a Multilayer Perceptron (MLP) model or liver patients with Benign End-Stage Liver Diseases (BESLD) and compared the performance of model with MELD and Sequential Organ Failure Assessment (SOFA) score. They collected 360 patient's data from the West China Hospital of Sichan University between February 1999 and August 2009 [9]. In order to overcome the slow performance and time consuming in training of back propagation networks, Cruz et al. [10] conducted a study with Radial Basis Function (RBF) networks using multi-objective evolutionary algorithm (MPENSGA2) in 2013. In order to match the donor-recipient pairs, they used a rule based system and fitness functions were used to train RBF networks [10]. They collected the data for the study from eleven Spanish hospitals. WEKA software was used for the classification of results [10]. These related research shows that the predictive models are capable of using live data of liver patients and capable of doing both diagnostic and predictive tasks [11]. Because of the simplicity in structure, ability to do parallel processing tasks, having long term memory, having fault tolerant ability and getting collective output, ANN models are better than logistic regression models [3].

\section{Materials and Methods}

\section{Dataset}

With the continuous exploration of rich dataset for the experimentation of prediction of survival of patients after liver transplantation resulted in the availability of United Nations Organ Sharing (UNOS) dataset. The UNOS is a tax-exempt, medical, scientific, and educational organization which controls the national Organ Procurement and Transplantation Network (OPTN) under agreement to the Division of Organ Transplantation (DOT) of Department of Health and Human Services (DHHS) [12].

The UNOS dataset is a multi-organ dataset since 1st October, 1987, from which we extracted the liver patient records. The dataset also consists of male and female liver patient records. Previous studies show that, they conducted study with either donor details, recipient details or transplantation details. But our study comprises donor details, recipient details and transplantation details which are shown in Table 1.

\begin{tabular}{|c|c|c|c|}
\hline Input attributes & Description of input attributes & Type of Attributes & Composite Attributes \\
\hline AGE_DON & Donor Age in Years & Numeric & \multirow{10}{*}{ Donor } \\
\hline CLIN_INFECT_DON & Deceased Donor-Clinical Infection $(\mathrm{Y}, \mathrm{N})$ & Nominal & \\
\hline CREAT_DON & Deceased Donor-Terminal Lab Creatinine & Numeric & \\
\hline DIABETES_DON & Deceased Donor-History of Diabetes $(\mathrm{Y}, \mathrm{N})$ & Nominal & \\
\hline DON_TY & Donor Type (Deceased, Living) & Nominal & \\
\hline GENDER_DON & Donor Gender & Nominal & \\
\hline NON_HRT_DON & Deceased Donor-Non-Heart Beating Donor & Nominal & \\
\hline SGOT_DON & Deceased Donor-Terminal SGOT/AST & Numeric & \\
\hline SGPT_DON & Deceased Donor-Terminal SGPT/ALT & Numeric & \\
\hline TBILI_DON & Deceased Donor-Terminal Total Bilirubin & Numeric & \\
\hline BMI_TCR & Body Mass Index of Recipient & Numeric & \multirow{13}{*}{ Recipient } \\
\hline ENCEPH_TCR & Recipient Encephalopathy & Nominal & \\
\hline EXC_HCC & $\begin{array}{l}\text { Type of Exception relative to HCC:HBL (HCC,NON-HCC: } \\
\text { HBL=Hepatoblastoma) }\end{array}$ & Nominal & \\
\hline FINAL_ALBUMIN & Most recent recipient Albumin & Numeric & \\
\hline FINAL_ASCITES & Most recent recipient Ascites & Numeric & \\
\hline FINAL_BILIRUBIN & Most recent recipient Bilirubin & Numeric & \\
\hline FINAL_INR & Most recent recipient INR & Numeric & \\
\hline FINAL_MELD_OR_PELD & Most recent recipient use MELD/PELD & Nominal & \\
\hline FINAL_MELD_PELD_LAB_SCORE & Most recent recipient MELD/PELD Lab score & Numeric & \\
\hline FINAL_SERUM_CREAT & Most recent recipient Serum Creatinine & Numeric & \\
\hline FINAL_SERUM_SODIUM & Most recent Serum Sodium & Numeric & \\
\hline GENDER & Recipient Gender & Numeric & \\
\hline INIT_AGE & Recipient Age in Years & Numeric & \\
\hline
\end{tabular}




\begin{tabular}{|c|c|c|c|}
\hline MALIG_TCR & Recipient any known Malignancies & Nominal & \\
\hline MALIG_TRR & Recipient malignancies at Transplantation & Nominal & \\
\hline GSTATUS & Graft status & Nominal & \\
\hline NUM_PREV_TX & The number of previous transplants & Numeric & \multirow[t]{2}{*}{ Transplantation } \\
\hline TXLIV & Type of liver(W,S ) & Nominal & \\
\hline
\end{tabular}

Table 1: Description of dataset to predict the patient survival using MLP.

The UNOS dataset consists of 65535 liver patient records with 389 variables since 1st October 1987 onwards. The MELD was proposed by Mayo clinic in the year 2002. As the study is based on MELD records, the records before 2002 and Pediatric End Stage Liver Disease (PELD) records were removed from the dataset.

Thus the resultant dataset comprises 383 liver patient records. The input parameters were represented in both numeric and nominal forms. The age of donor, AGE_DON and age of recipient, INIT_AGE played an important role in predicting the survival of liver patients after liver transplantation [13].

The donor parameters used in the study include CREAT_DON, SGOT_DON, SGPT_DON and TBILI_DON were represented as numeric. CLIN_INFECT_DON represents the donor have any type of infection or not. CLIN_INFECT_DON were represented as $(\mathrm{N}=\mathrm{No}$, $\mathrm{Y}=$ Yes, $\mathrm{U}=$ Unspecified). DIABETES_DON represents the donor is having the history of diabetes or not. DON_TY used for representing the type of donor.

Two type of donors were used which includes Deceased donors and Living donors. We experimented our model with 352 deceased donors and 31 living donors. GENDER_DON represents the gender of donors. Our study includes 154 female donors and 229 male donors.

GENDER indicates the recipient gender which includes 145 female recipients and 238 male recipients. The non-heart beating donors in the study were represented by NON_HRT_DON. The attribute BMI_TCR is the BMI (Body Mass Index) of recipient which is represented as numeric attribute.

$\mathrm{BMI}$ is the ratio of weight of patient to the square of height of the patient [14]. ENCEPH_TCR is the encephalopathy of recipient which is a nominal attribute represented as $\mathrm{YNU}$ ( $\mathrm{Y}=\mathrm{Yes}, \mathrm{N}=\mathrm{No}$, $\mathrm{U}=$ Unspecified).

The dataset includes recipients containing HCC and non HCC denoting the attribute EXC_HCC. FINAL_ALBUMIN, FINAL_ASCITES, FINAL_BILIRUBIN AND FINAL_INR are clinical attributes of recipients for Albumin, Ascites, Bilirubin and INR values. INR in the MELD score replaces Prothrombin Time (PT) and is measured with respect to appropriate ISI of the local PT test system.

$$
\mathrm{INR}=(\text { Patient's PT } / \text { MNPT })
$$

MNPT is calculated as the geometric mean of PT of at least 20 adult normal subjects of both sexes. The MELD score is calculated by the formula,

MELD Score $=9.6^{*} \log _{\mathrm{e}}[$ creatinine $\mathrm{mg} / \mathrm{dl}]+3.8^{*}\left(\log _{\mathrm{e}}[\right.$ bilirubin $\mathrm{mg} / \mathrm{dl}]+11.2^{*} \log _{\mathrm{e}}[\mathrm{INR}]+6.4^{*}$ cause of cirrhosis [0 if alcoholic or cholestatic liver disease, 1 if otherwise) (2),

\begin{tabular}{|l|l|}
\hline MELD VALUE & RESULTS \\
\hline MELD $<15$ & Best \\
\hline MELD,15-25 & Good \\
\hline MELD $>25$ & More complicated \\
\hline MELD $>40$ & Bad \\
\hline
\end{tabular}

Table 2: Analysis of performance of MELD score.

Clinicians performed the analysis of MELD score according to the performance as shown in Table 2. The survival chance of the liver patients is very poor with MELD>40 [15]. Low survival rates results in MELD $>25$ and good survival rates achieved with MELD, 15-25 [15]. Doctors give higher preference to patients with MELD $<15$ for liver transplantation for getting maximum survival rates [15]. FINAL_SERUM_CREAT and FINAL_SERUM_SODIUM are the final serum creatinine and final serum sodium attributes of recipient. Whether the patients are having any other malignancies or not are represented by MALIG_TCR. MALIG_TCR, a nominal attribute also represented as YNU ( $\mathrm{Y}=\mathrm{Yes}, \mathrm{N}=\mathrm{No}, \mathrm{U}=\mathrm{Unspecified).} \mathrm{MALIG \_ TRR}$ represents the recipient having malignancies at transplantation. NUM_PREV_TX denotes the patients undergone any other transplantation or not represented by numeric attribute. TX_LIV denotes the type of liver represented by two variables such as $\mathrm{W}$ for Whole liver and $\mathrm{S}$ for Split liver.

The input parameters were given to the MLP model to train the data for predicting the survival of liver patients after liver transplantation. GSTATUS is the output of the model which was represented as binary. The best survival of graft of liver patients after liver transplantation with GSTATUS $=0$ and poor survival of graft of liver patients after liver transplantation with GSTATUS=1. This model put forward a lot of merits over conventional statistical techniques with the selection of suitable dataset.

\section{Classifiers in survival prediction}

We used three classifiers to prove the accuracy of survival prediction in liver transplantation patients. The classifiers such as Multilayer Perceptron, RBF and Adaptive Resonance Theory (ART) MAP were used in survival prediction by training the dataset and compared each other according to various performance measures. WEKA software is used to train the classifiers and the output of the classifiers is shown as Receiver Operating Characteristics (ROC) curves [16]. 


\section{MLP classifier}

The dataset consisting 27 attributes were given to the MLP model, train the data and output taken was GSTATUS. The MLP classifier trains the data using Back propagation algorithm. We passed the input attributes to the input layer of MLP model. Donor-recipient matching was done in the hidden layer of MLP classifier. With 10 fold cross validation; best output was taken from the classifier which was considered as GSTATUS. The activation function used to train the data was sigmoid activation functions. With multiple hidden layers, we could reduce errors while training the data. Out of three classifiers, MLP classifier showed higher accuracy of $99.74 \%$ than other classifiers.

\section{RBF classifier}

RBF is a feed forward ANN which consists of three layers including input layer, hidden layer and output layer. As the dataset consists of both numerical and nominal data, we have chosen a normalized Gaussian Radial Basis Function network for training the data. Kmeans clustering algorithm was applied to the hidden layer in the RBF classifier. The classifier trained with nonlinear functions in the hidden layer and linear functions in the output layer. Logistic regression was used with nominal class problems and linear regression was applied with numeric class problems. Symmetric Multivariate Gaussian (SMG) functions were used to fit the data from each cluster. SMG uses the set of clusters per class for the nominal attributes. The numeric attributes are standardized to zero mean and unit variance. The logistic regression was used to $\mathrm{K}$-means clusters as basic functions with ridge parameter of $1.0 \mathrm{E}-8$. The activation function used in RBF networks is radial monotonically increases or decreases with distance from the center [17]. While training the data we could observe that, the accuracy of RBF is lesser than MLP and better than ARTMAP. But the training time of RBF is faster than MLP.

\section{ARTMAP classifier}

We also trained the medical data with ARTMAP classifier. The recipient data and donor data are allocated in the comparison field and recognition field. The ARTMAP training is based on vigilance parameter [18]. The vigilance parameter in the ART networks acts as a threshold while comparing the donor as well as recipient data. According to the vigilance parameter, the best match was performed and the liver is allocated from the suitable donor. If the liver is in mismatch, the process repeats until the best match was found out. Out of different ART networks, we used ARTMAP 0 and compared it with MLP and RBF. Due to Stability-Plasticity nature of ART networks, the training is faster in ARTMAP than MLP and RBF. But the accuracy is far lesser than MLP and RBF.

\section{Prediction using MLP classifier}

The input data containing 27 attributes were given to the MLP model and the output node acted as GSTATUS. While training the clinical data, the algorithm used is Back propagation algorithm and activation function used in the hidden layers was sigmoid function. Proper donor-recipient matching is needed for the best survival of patients undergoing liver transplantation. This is done with the help of hidden layers during training the model. The model is trained at a rate of 0.3 . As the numbers of hidden layers are increased, the training time also increased and thus the errors were reduced. The errors could be minimized by adjusting the weights for a best survival. In our model, the momentum of 0.2 was applied. In order to avoid weight oscillations in the hidden layer of model, instability and network adaptability, the momentum factor should be kept less than one [19]. Since the output attribute, GSTATUS is binary, linear function was applied from hidden layer to the output layer. The complexity of the model, classification accuracy, training time and the various model performance measures were the features taken in the training phase. In our model, the total number of epochs used is 1500 . During each epoch of training, the classification accuracy was computed and produced the best survival output. By increasing the number of epochs, mismatch between donor and recipient were minimized.

If the GSTATUS $=0$ represents the Graft survived which shows the best survival. If GSTATUS=1 represents the Graft failed which shows the poor graft survival.

\section{Model evaluation criteria}

$\mathrm{k}$-fold cross-validation: In k-fold cross validation, the total dataset can be split randomly into $\mathrm{k}$ mutually exclusive folds of equal size, (DS1, DS2,..., DSk). The formula to calculate the cross validation (CV) accuracy is given as,

$$
\mathrm{CV}=\frac{1}{\mathrm{k}} \sum_{\mathrm{i}=1}^{\mathrm{k}} \mathrm{Ai}[19] \text { (3) }
$$

From equation (3), Ai is each fold accuracy and $\mathrm{k}$ is the number of folds. This means that the entire dataset is trained and tested $\mathrm{k}$ number of times. We used 10 fold cross validation procedure with 10 equal numbers of folds for the whole dataset. We also used stratified cross validation procedure in which each fold contains the same proportion of predictor labels as the original dataset.

Accuracy performance measures: In order to evaluate the performance of MLP model, there were some measures used in the system. The various performance measures determined are Sensitivity, Specificity and Accuracy [20]. Along with the performance measures, various performance error measures like Mean Absolute Error (MAE), Root Mean Squared Error (RMSE), Relative Absolute Error (RAE) and Root Relative Squared Error (RRSE) were evaluated. The MAE and RAE were reduced with increase in number of hidden layers. The RMSE and RRSE had slightly improved with increase of hidden layers. But RMSE remained constant with decrease of MAE and RAE. RRSE reduced with increase of learning rate.

\section{Results and Discussion}

\section{Role of parameters and survival prediction}

We ranked all the input attributes used to train the model using WEKA software. We found out with weights that the entire donor attributes, recipient attributes and transplantation attributes are having equal importance with ranking.

Depends upon the pre transplantation state of the recipient, the availability of donor and surgery complications, the post transplantation outcome of patient may vary [21]. Without any difficulty, 243 recipients out of 383 were alive after liver transplantation. 34 recipients out of 243 were survived through re transplantation. Again 24 recipients lost their graft. But 22 recipients out of 24 were survived through re transplantation. 2 recipients were dead. A total of 116 recipients were dead immediately after transplantation initial itself. The donor age and recipient age were represented as numeric. So after training, the mean and standard 
deviation for the donor and recipient age are $40.88 \pm 17.49$ and $45.16 \pm$ 11.77. Gender mismatched liver transplants lead to poor survival rate [22]. The best survival rate will result in transplantation with female recipient from a male donor [22]. Poor survival results in male recipient from a female donor [22]. While taking the parameter infection, it was noticed that, 129 donors were affected with infection. We got the mean and standard deviation value of creatinine as $1.33 \pm$ 1.33. There were 31 living donor grafts and out of that 7 living donor grafts were lost after transplantation. In the deceased donors, 344 were non-heart beat donors and 8 were heart beat donors with a missing value of 31 heart beat or non-heart beat donors. In our dataset, 315 donors were not having diabetes and 37 donors were having diabetes with a missing of 31 missing donor records. After transplantation, 8 donor grafts out of 37 donor grafts were lost. The mean and standard deviation of SGPT and SGOT were represented as 76.07 \pm 158.94 and $86.45 \pm 161.08 .38$ patient records were missing in SGOT and again 38 liver patient records were missing in SGPT.

The Total Albumin value we got as $0.89 \pm 0.72$ in the place of reference range 3.4-5.4 g/dL. There are total 39 records is missing in Total Albumin data. 97 recipients were having encephalopathy and 255 recipients were not having encephalopathy. 12 patients' record was missing in encephalopathy data. In the case of HCC, 97 liver patients were suffering with HCC and 286 recipients were not having HCC. The mean and standard deviation of Final_Albumin, Final_Ascites, Final_Bilirubin, INR, Serum Creatinine and Serum Sodium were are $2.92 \pm 0.71,1.94 \pm 0.7,7.28 \pm 10.301,1.793 \pm 1.625,1.43 \pm 1.246$ and $136.2 \pm 6.02$. In the dataset, 13 liver patients were having malignancies and 339 liver patients were not having malignancies. 9 records were missing and 22 liver patients' records were in the unspecified format. 347 liver patients were undergone whole liver transplantation and 36 liver patients were undergone split liver transplantation. We could predict the survival of liver patients after liver transplantation with proper dataset and model and produced the result with $99.74 \%$ accuracy.

\section{Performance evaluation of MLP model}

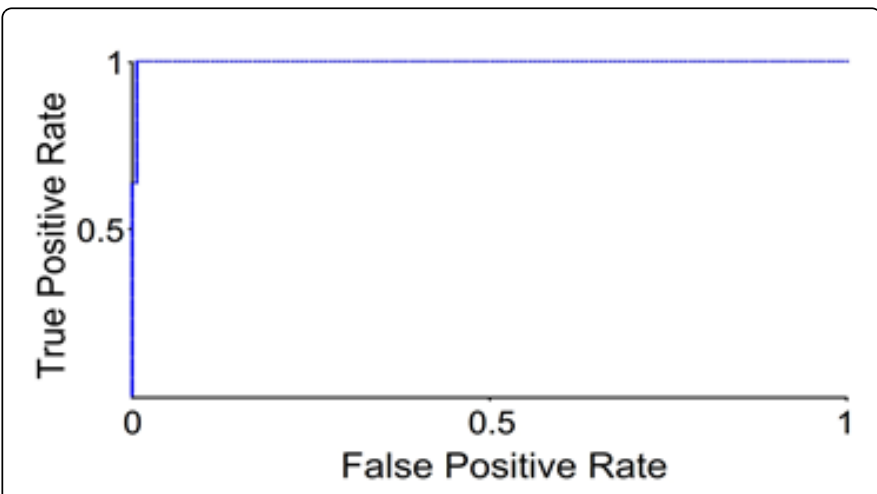

Figure 1: ROC curve of proposed MLP model.

We evaluated the performance measures of MLP model with the help of ROC curves using WEKA software. While drawing the ROC curves, the $\mathrm{X}$-axis is the False Positive Rate and Y-axis is the True Positive Rate which is shown in Figure 1. With the training of 383 liver patient records, the True Positive Rate achieved is 0.007 and the False Positive Rate is 1 . The time taken to build the model is $16.97 \mathrm{~s}$. The survival accuracy obtained while training the model is $99.74 \%$.

\section{Comparison of proposed MLP with proposed RBF and ARTMAP}

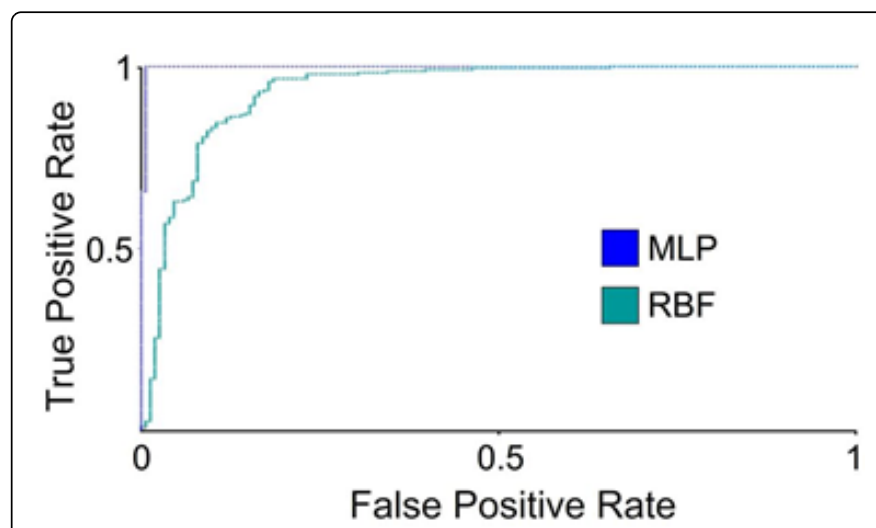

Figure 2: Comparison between proposed MLP model and RBF model.

We trained the same dataset in our RBF and ARTMAP models and compared those performances with proposed MLP. We obtained the accuracy of proposed RBF as $86.95 \%$ and proposed ARTMAP as $54.05 \%$. But the accuracy of proposed MLP is $99.74 \%$. From this, it is clearly observed that the accuracy of proposed MLP is better than proposed RBF and ARTMAP. The time taken to build MLP model is $16.97 \mathrm{~s}$. But the time taken to build the RBF model and ARTMAP model are $0.32 \mathrm{~s}$ and $0.98 \mathrm{~s}$. The MLP model training takes too much time than RBF and ARTMAP models. The Area under Curve (AUC) of MLP is 0.9975. But the AUC of RBF and ARTMAP are 0.928 and 0.498 . Researchers proved that the ANN models with AUC more than 0.5 can be used for prediction purposes. Thus we can choose MLP and RBF for prediction purposes. We also experimented and evaluated the various performance error measures between different ANN models which are shown in Table 3. Because of high accuracy, MLP is more suitable than other models for the prediction of survival after LT as shown in Figure 2.

\section{Comparison of proposed MLP with existing approaches}

From the dataset, the total patients survived based on MELD score is 303 and 80 liver patients were not survived. So the survival rate based on MELD score is $79.11 \%$. From the Zhang et al. [9] study, we could observe that the first year survival accuracy is $89.7 \%$ and second year survival accuracy is $86.2 \%$. Their study includes data of 360 liver patients with Benign End stage liver disease. Cucchetti et al. [7] proposed an ANN with survival accuracy $82 \%$ to $91 \%$. Their dataset includes 251 patients with cirrhosis. With the appropriate selection of model and dataset, we achieved a survival accuracy of $99.74 \%$ using MLP model for the liver transplantation patients. 


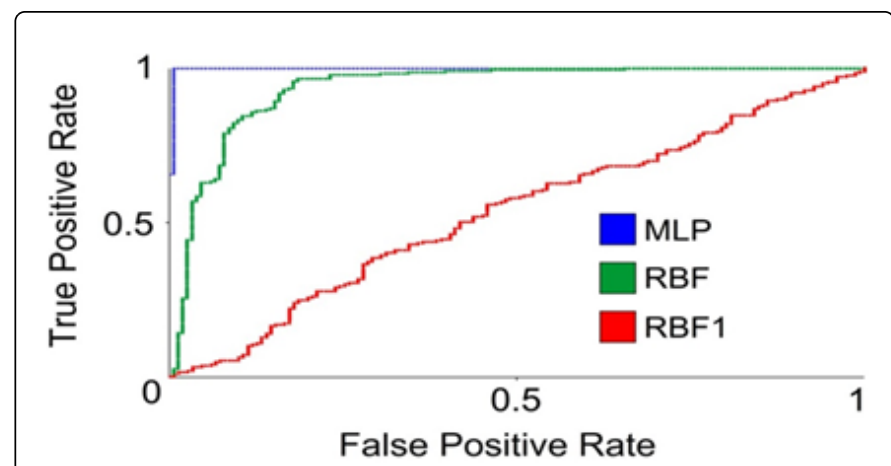

Figure 3: Comparison of proposed models with existing model.

\begin{tabular}{|c|c|c|c|c|c|}
\hline $\begin{array}{l}\text { Evaluation measures of } \\
\text { Classifiers }\end{array}$ & $\begin{array}{l}\text { Output Prediction Based } \\
\text { on }\end{array}$ & Values Obtained with MLP & $\begin{array}{l}\text { Values Obtained with } \\
\text { RBF }\end{array}$ & $\begin{array}{l}\text { Values } \\
\text { ARTMAP }\end{array}$ & with \\
\hline \multirow[t]{3}{*}{ Performance Measures } & TP rate & 0.007 & 0.862 & 0.698 & \\
\hline & FP rate & 1 & 0.119 & 0.702 & \\
\hline & Accuracy \% & $99.74 \%$ & $86.95 \%$ & $54.05 \%$ & \\
\hline \multirow[t]{4}{*}{ Performance Error Measures } & MAE & 0.0049 & 0.1649 & 0.4595 & \\
\hline & RMSE & 0.0049 & 0.3018 & 0.6779 & \\
\hline & RAE\% & $1.03 \%$ & $34.51 \%$ & $96.18 \%$ & \\
\hline & RRSE\% & $10.78 \%$ & $61.76 \%$ & $138.71 \%$ & \\
\hline Time taken in seconds & & $16.97 \mathrm{~s}$ & $0.32 \mathrm{~s}$ & $0.98 \mathrm{~s}$ & \\
\hline
\end{tabular}

Table 3: Performance Evaluation results of MLP model for three month survival prediction with RBF and ARTMAP. P rate: True Positive rate, FP rate: False Positive rate, MAE: Mean Absolute Error, RMSE: Root Mean Squared Error, RAE: Relative Absolute Error, RRSE: Root Relative Squared Error, MLP: Multilayer Perceptron, RBF: Radial Basis Function, ARTMAP: Adaptive Resonance Theory MAP.

Cruz et al. [10] proposed RBF using genetic algorithm (RBF1) for the prediction of liver transplantation survival. Normally, genetic algorithms are used for multidimensional problems; it is difficult to find out a global optimum. We compared our RBF model with RBF1 and found out that proposed RBF model could perform more with 0.928 AUC than RBF1 which is shown in Figure 3. The AUC of RBF1 is 0.5659 which is lower than proposed RBF model. As the ANN can overcome the local minima, it is suitable to perform nonlinear problems. Thus it is clearly stated that ANN models are better to predict the survival of patients after liver transplantation.

\section{Conclusion}

Liver transplantation is the best and accurate solution for the end stage liver disease. The most important aspects of liver transplantation are the organ collection and sharing. Now a day's, liver transplantation is one of the challenging areas in the field of organ transplantation. Human liver get injured due to not only alcohol or liver disease but also it affects by improper food habit as well as genetic syndromes. Clinical studies indicated that in the next epoch, more than $90 \%$ people will be affected by liver complications. Presently the medical experts predict the survival of patients after liver transplantation is based on MELD score. As the creatinine varies with body weight of the liver patient and it is lower in females than males, a group of researchers introduced ANN models to predict the patient survival after liver transplantation. Based on our dataset, the total liver patients survived are based on MELD score is $79.11 \%$ and failure percentage is $20.89 \%$. Through this study, we could achieve a high accurate survival prediction with $99.74 \%$ with the proper selection of ANN model and dataset. We compared the proposed MLP with other classifiers such as proposed RBF and ARTMAP. We also could compare the proposed RBF model with existing RBF model. With all these findings, we could evaluate that MLP is best suitable ANN model to predict the three month survival of patients after liver transplantation.

\section{Ethical Approval and Consent}

The data was collected based on OPTN data as of 5th June 2015. This work was supported in part by Health Resources and Services Administration contract 234-2005-370011C. The content is the responsibility of the authors alone and does not necessarily reflect the views or policies of the Department of Health and Human Services, nor does mention of trade names, commercial products, or organizations imply endorsement by the US Government. 
Citation: Raji CG, Chandra SSV (2016) Artificial Neural Networks in Prediction of Patient Survival after Liver Transplantation. J Health Med Inform 7: 215. doi:10.4172/2157-7420.1000215

Page 7 of 7

\section{Acknowledgement}

We would like to express our sincere thanks to Dr. Arun Kumar M.L, MS, MCh, MRCS Ed, PDF (HPB), Associate Professor and Chief Surgeon in Gastro Enterology, Hepato Biliary and Pancreatic Surgery and LT, Sree Gokulam Medical College and Research Foundation, Thiruvananthapuram, India for the valuable technical support including comments and suggestions to improve the quality of the study. We are also thankful to the authorities of Medical Colleges in Kerala State for sharing this valuable information in organ transplantation.

\section{References}

1. Merion, Robert M, Schaubel DE, Dykstra DM, Freeman RB, et al. (2005) The survival benefit of liver transplantation. Am J of Transplant 5: 307-313.

2. Kamath PS, Wiesner RH, Malinchoc M, Kremers W, Therneau TM, et al (2001) A model to predict survival in patients with end-stage liver disease. Hepatology 33: 464-470.

3. Chandra V, Reshmi G, Achuthsankar S, Sreedharan S, Radhakrishanan M (2010) MTar: a computational microRNA target prediction architecture for human transcriptome. BMC bioinformatics 11 Suppl 1: S2.

4. Doyle HR, Ignazio RM, Jabbour N, Zetti G, McMichael J, et al. (1994) Early death or retransplantation in adults after orthotopic liver transplantation: Can Outcome be predicted? 1." Transplantation 57: 1028.

5. Doyle HR, Dvorchik I, Mitchell S, Marino IR, Ebert FH, et al. (1994) Predicting outcomes after liver transplantation. A connectionist approach. Ann Surg 219: 408-415.

6. Parmanto B, Doyle HR (2001) Recurrent neural networks for predicting outcomes after LT: representing temporal sequence of clinical observations." Methods Inf Med 40: 386-391.

7. Cucchetti A, Vivarelli M, Heaton ND, Phillips S, Piscaglia F, et al. (2007) Artificial neural network is superior to MELD in predicting mortality of patients with end-stage liver disease. Gut 56: 253-258.

8. Marsh JW, Dvorchik I, Subotin M, Balan V, Rakela J, et al. (1997) The prediction of risk of recurrence and time to recurrence of hepatocellular carcinoma after orthotopic liver transplantation: a pilot study. Hepatology 26: $444-450$

9. Zhang M, Yin F, Chen B, Li YP, Yan LN, et al. (2012) Pretransplant prediction of posttransplant survival for liver recipients with benign endstage liver diseases: a nonlinear model. PLoS One 7: e31256.

10. Cruz RM, César HM, Juan CF, Javier B, Manueldel M (2013) Predicting patient survival after LT using evolutionary multi-objective artificial neural networks. Artificial intelligence in medicine 58.1: 37-49.

11. Dreiseitl S, Machado OL (2002) Logistic regression and artificial neural network classification models: a methodology review. Journal of biomedical informatics 35: 352-359.

12. Oztekin, Asil, Dursun D, Zhenyu JK (2009) Predicting the graft survival for heart-lung transplantation patients: An integrated data mining methodology. international journal of medical informatics 78.12: e84-e96.

13. Busuttil RW, Tanaka K (2003) The utility of marginal donors in liver transplantation. Liver Transpl 9: 651-663.

14. Calle EE (1999) Body-mass index and mortality in a prospective cohort of US adults." New England Journal of Medicine 341:1097-1105.

15. Poller L (2004) International Normalized Ratios (INR): the first 20 years. J Thromb Haemost 2: 849-860.

16. Chandra V, Hareendran A (2014) Artificial Intelligence and Machine Learning.

17. Orr, Mark JL (1996) Introduction to radial basis function networks.

18. Yang, Bo Sk, Tao H, JL art (2004) An "ART-KOHONEN neural network for fault diagnosis of rotating machinery." MechanicalSystems and Signal Processing 18: 645-657.

19. Saduf, Wani (2013) M.: Comparative study of back propagation learning algorithms for neural networks. International Journal of Advanced Research in Computer Science and Software Engineering.

20. Vinod Chandra SS, Reshmi G (2009) A pre-microRNA classifier by structural and thermodynamic motifs." Nature \& Biologically Inspired Computing. NaBIC 2009.

21. Moreno R, Berenguer M (2006) Post-liver transplantation medical complications. Ann Hepatol 5: 77-85.

22. Lai JC, Feng S, Roberts JP, Terrault NA (2011) Gender differences in liver donor quality are predictive of graft loss. Am J Transplant 11: 296-302. 